\title{
HCI and Information Search: Capturing Task and Searcher Characteristics Through 'User Ability to Specify Information Need'
}

\author{
Naresh Kumar Agarwal and Danny C.C. Poo \\ School of Computing, National University of Singapore \\ 3 Science Drive 2, Singapore 117543 \\ \{naresh, dpoo\}@comp.nus.edu.sg
}

\begin{abstract}
Human-Computer Interaction (HCI) is all about the way in which people interact with computer systems. This paper focuses on the cognitive aspects of HCI when a user is searching for information, so as to facilitate effective user interactions with vast amounts of available information. Search engines provide a 'one-size-fits-all' model, which do not adequately cater to the differing needs of searchers at different points in time (continuously changing situations in time/space, as per sense-making theory). We posit that from a system designer's point of view, capturing the 'user ability to specify his information need' will help operationalize task/searcher characteristics (hence the user need) and help the designer provide better interfaces for search that fit the needs of the user and lead to search efficacy and searcher satisfaction. The study should advance HCI for search through greater understanding of user needs, enhance search interfaces and lead to theory development.
\end{abstract}

Keywords: Human-computer interaction for search, sense-making theory, tasktechnology fit, information searching and retrieval.

\section{Introduction}

Human-Computer Interaction (HCI) is all about the way in which people (individuals, groups or organizations) interact with computer systems [1]. HCI involves physical aspects of the interaction (are the keys spaced right?), perceptual aspects (is the text color easy to see against the background?), cognitive aspects (will these menu names be understood?), and social aspects (will people trust each other on this auction site?) [1]. This paper focuses on the cognitive aspects of human-computer interaction when a user is searching for and retrieving information, so as to facilitate effective user interactions with the vast amount of information available information today.

\subsection{Limitations of Current Search Interfaces}

Search engines have become the gatekeepers to online information by helping people find information [2]. However, no single search engine indexes the entire WWW (maximum coverage estimated to be $69.6 \%$ as of 2005 [3]). Search engines don't perform well when two search words have the same meaning or a single word has 
multiple meanings [4,2]. Effective query formulation is possible only when the users are already familiar with the research topic and can see the subtle differences in its vocabulary [5] i.e. it depends on the user's ability to specify information need (which in turn depends on the characteristics of the task and the searcher). Also, the interests of the users vary with time (Dervin [6], as per her sense-making approach, calls them continuously changing situations in time/space) and cannot be represented by a fixed set [7] or a 'one-size-fits-all' model widely prevalent in the search engines of today. Thus, there is a lack of 'fit' between the information systems available for search and the task needs of different searchers or of the same searcher at different times or different stages of the information search process.

Knowledge discovery can happen if information systems are designed which take into the account the searcher's differing abilities to specify information need (depending on the complexity of the task, prior domain knowledge, etc.) and provide technology features that match the task and searcher characteristics reflected from this ability. Designers of interfaces for search must strive to design interfaces that capture the unique context of the searcher (his task and searcher characteristics, which define his need). While this may be easier said, this is a humongous task, as the needs of a searcher reside in his head and change continuously (sense-making theory). Different tools and technology characteristics have been developed to facilitate different aspects of searching behavior. However, the efficacy of these tools may depend on the user's differing ability to specify their information need.

In this paper, we describe how from a system designers point of view, capturing the user ability to specify his information need, will help operationalize task and searcher characteristics (hence the user need) and help a system designer provide better interfaces for search, which fit the needs of the user and lead to search efficacy and searcher satisfaction. The study should advance HCI for search through greater understanding of user needs, enhance search interfaces and lead to theory development.

In the next section, we discuss calls for collaboration between the person-centric information seeking studies and the system-centric information retrieval studies. We also look at the two theories that provide the theoretical basis for this study. In Section 3, we look at task, searcher characteristics and need, and explain the difficulty in capturing a user's information need. In Section 4, we look at the searcher's ability to specify information need, which can be used to operationalize task and searcher characteristics. Section 5 concludes the paper and mentions future work to be done.

\section{Need to Bridge 'System-Centric' and 'User-Centric' Studies}

The tradition of research into information seeking considers information seeking from a systems perspective and information users as passive, situation independent receivers of objective information [8]. Yet it has been often accepted that information needs and information seeking processes depend on user's tasks $[9,10,11]$. Thus, many studies have investigated the relationships of various types of tasks and information seeking behavior [12]. 
Since the early 1980's, there has been a movement away from system-centeric studies to person or user-centered studies [13]. Person-centered research offers understanding of information seeking and use within the various contexts of people's lives. Important meta-theories (e.g. Dervin's sense-making [14]) and models (e.g. Wilson's model of information seeking [15], Information Search Process [16], etc.) have been developed. New ways of looking at information seeking have emerged, such as Savolainen's [17] work on Everyday Life Information Seeking. However, transfer of concepts across user studies and information retrieval/information systems remains problematic and insufficient [18]. Kuhlthau [18] has called for collaboration between the insights of user studies and the innovations of information retrieval and information systems. Ingwersen and Jarvelin [19,20] have also concluded that Information Retrieval research needs extension toward more context and Information Seeking research needs extension towards task and technology. Taking Kuhlthau's [18], Ingwersen's and Jarvelin's [19,20] call, this study endeavors to work towards a 'fit' between the needs of the information searcher (user studies/information seeking) and the services provided by the information system (information retrieval) through the user ability to specify his information need.

The study draws its theoretical basis from two theories - the Task-Technology Fit Theory and the Sense-Making Theory.

\subsection{Task-Technology Fit Theory}

The ability of IT to support a task is expressed by task-technology fit (TTF), which implies matching the capabilities of the technology to the demands of the task [21]. As shown in Fig. 1, a match between the characteristics of the task and the searcher (including his needs) and the characteristics of the technology will lead to a tasktechnology fit, which will result in increased search precision and a higher level of searcher satisfaction.

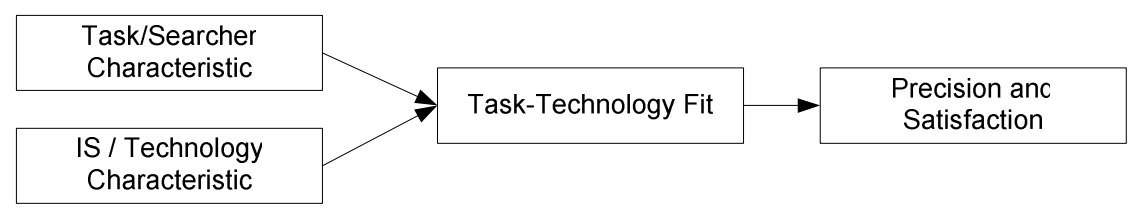

Fig. 1. TTF model adapted for Information Search and Retrieval

Kuhlthau [22,18], in his study of the information search process of lawyers, observed that the lawyers' experiences and expectations in complex information seeking tasks were not easily accommodated by the information system available to them. Similarly, Van Ossenbruggen and Hardman [23] observed that though general purpose search engines are widely prevalent, their focus on providing a one-size-fits all model for search leads to inadequate search results. Thus, it is imperative that the characteristics of the information system match the needs of the searcher and his search task. 


\subsection{Sense-Making Paradigm}

Using Brenda Dervin's [6] sense-making paradigm, we propose that task and searcher characteristics can be operationalized as the searcher's ability to specify information need. According to the paradigm, the need of a searcher is unique only in a situation in time-space and keeps changing. The theory proposes that information is not 'something that exists apart from human behavioural activity.' Rather, it is 'created at a specific moment in time-space by one or more humans' [6 p.63]. Unlike other approaches to information seeking that see information as something 'out there' that is transmitted to people (as Dervin says, an information 'brick' that is put into a human 'bucket'), sense-making sees information as construed internally in order to address gaps or discontinuities [24,15].

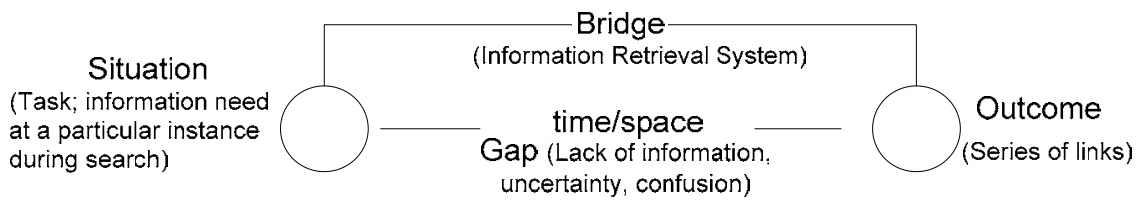

Fig. 2. Dervin's model (adapted from [15])

Sense-making is implemented in terms of four constituent elements: a situation in time and space, which defines the context in which information problems arise; a gap, which identifies the difference between the contextual situation and the desired situation (e.g. uncertainty); an outcome, that is, the consequences of the sense-making approach, and a bridge, i.e. some means of closing the gap between situation and outcome [15] (see Fig. 2). Tools are needed to support continuous sense-making. An effective way for the information system to accommodate the context of the search (searcher and task characteristics) is to understand how well the searcher is able to specify his need before the IS, and develop technology features pertaining to that.

\section{Difficulty in Capturing User Need and Task/Searcher Characteristics}

Having looked at the two major theories underlying this study, let us investigate the difficulty in trying to capture the user need and his unique search context, based on the characteristics of the searcher and the task at hand.

\subsection{Task}

Kim and Allen [25] cite a number of empirical studies that have supported the premise that user's search performance and/or patterns differ depending on the task. Saracevic and Kantor [26] have found that the specificity and complexity (broad and specific questions) of search task have an impact on search performance. Several studies have used task with different levels of specificity to investigate the impact of 
tasks on search behavior - closed and open tasks [27], general and specific tasks [28], topical and factual tasks [29] and subject and known-item searches [30].

In the course of promoting a seven-step strategy to web searchers, Pffafenberger [31] divided tasks based on the amount of information needed for a topic into three types of questions: 1) Finding specific information 2) Collecting a few sources of high quality information 3) Collecting everything on a topic i.e. conducting an exhaustive search to retrieve all available material. Spool et al. [32] defined four types of questions: 1) Simple fact questions, simplest type with only one correct answer 2) Judgment questions, where user must locate as well as analyze potential answers 3) Comparison of fact questions, where the user researches two or more questions to arrive at an answer 4) Comparison of judgment questions, situations involving comparisons and judgments [33]. Kuhlthau [16] defines a 6-stage information search process and the feelings and thoughts common to each stage. A successive search process is implicit in Kuhlthau's analysis of the search activity [15].

Thus, different characteristics of task lead to different information need and questions that need answering. The tasks might also differ depending on which stage of the information seeking person the searcher is at.

\subsection{Searcher Characteristics}

Prior research has investigated different aspects of the searcher during information search:

- Individual differences. Aspects such as the user's cognitive abilities, cognitive style and problem-solving style [25]

- Information goal. Limberg [34] found that within the same assignment, the goals of fact-finding, getting a right answer or analyzing and synthesizing resulted in quite different outcomes. Information goal might explain the different approaches to information seeking by individuals with the same or similar task. Kuhlthau [22] found in her study that the expert (who was seeking to add value to the client's knowledge) had quite different goals in information seeking than the novice (who was looking for the right answer).

- System knowledge. System knowledge is the searcher's familiarity/expertise with the Information System and searching techniques [35].

- Domain knowledge. Domain knowledge is the searcher's knowledge of the search subject or topic. Miura, Fujihara and Yamashita [36] concluded that task-related domain-specific knowledge has a much greater impact on various stages of their retrieval behaviour (compared to knowledge relevant to search engines or browsing i.e. system knowledge).

The above characteristics, as well as factors like experience and prior success with search play an important role in determining the context and need of the search.

\subsection{Information Need}

'Need for information consists of the process of perceiving a difference between an ideal state of knowledge and the actual state of knowledge' [37 p.463]. Need involves reaching a desired goal [24]. Harter [38] argues that to talk about an individual's 
information need is virtually the same as describing his or her 'current psychological state', because needs shift stochastically as each relevant piece of information is encountered. One bit of knowledge may raise questions, lead to another fact or a new conclusion, and so forth, which changes one's knowledge state and hence what one finds relevant and worth seeking [24].

As per Dervin's [6] sense-making approach, new knowledge from query results (outcome) may raise questions, lead to another fact or a new conclusion (situation in time/space), which changes one's knowledge state (state). Dervin's use of time/space underlines the ever-changing nature of information need.

Belkin et al. [9]'s concept of need is that of an 'anomalous state of knowledge' (ASK). An ASK exists when a person recognizes that there is an anomaly (gap or uncertainty) in their state of knowledge regarding a situation or topic. He may then try to address the anomaly by requesting for information. He will then judge if the anomaly has been resolved. If it is not resolved, another ASK may be generated, or the motivation to address it may be exhausted. Case [24] interestingly points out that the searcher always 'gives up' eventually, because there is always more that could be known regarding a topic. The question of 'when' is determined by available resources and the searcher's level of motivation. Thus, the searcher's need is a complex context consisting of the perceived work task or interest as well as perceptions and interpretations of: knowledge gap or ASK and relevance; uncertainty and other emotional states; the potential sources for the solution (if any) of the work task or interest; the intentionality i.e. goals, purposes, motivation, etc.; information preferences, strategies, pressures (costs, time); self i.e. own capabilities, health, experiences; systematic and interactive features and information objects [39].

From the discussion on information need above, it is evident that from the information retrieval system's perspective, it is extremely difficult to pin-point the user's information need at a particular time/space and to provide compatible technology features to address this need. 'Typically, the cost of acquiring full context is simply too high, compared to the benefits, let alone possible privacy issues' [40].

However, even though the searcher's information need cannot be adequately captured by the information system, what is possible to capture is the searcher's ability to specify information need. It is also possible to be unaware of one's true need [41]. At such times, a searcher is often looking for the right questions or the right keyword to ask, rather than the answer to the need right away.

\section{Ability to Specify Information Need}

The searcher's ability to specify information need is defined as the state resulting from task and searcher's characteristics which determines how effectively the searcher is able to interact with the information system to retrieve information pertaining to the need.

The same person may exhibit different abilities to specify information need depending on the complexity/unstructuredness of the task he is working on and the domain knowledge he has in the area of search. However, two different people will have different abilities to specify information need depending on their individual 
Table 1. Effect of Task and Searcher Characteristics on the ability to specify information need

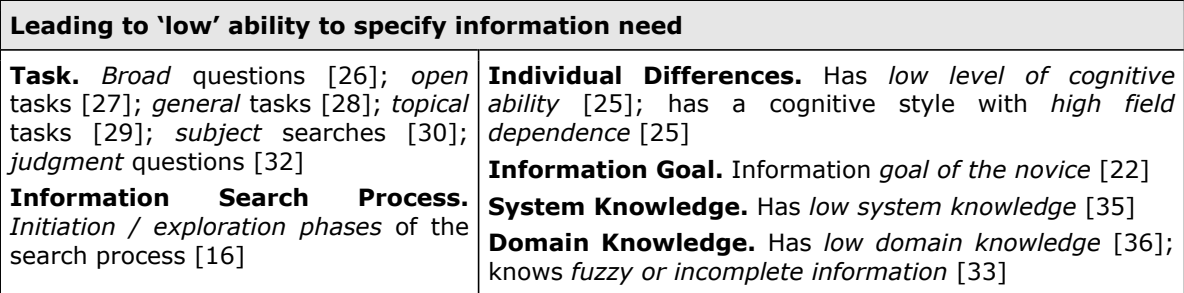

Leading to 'high' ability to specify information need

Task. Specific questions [26]; closed Individual Differences. Has high level of cognitive tasks [27]; specific tasks [28]; factual ability [25]; has a cognitive style with high field tasks [29]; known-item searches independence [25]

[30];

simple fact questions [32]

Information Search Process.

Formulation / collection phases of the search process [16]

Information Goal. Information goal of the expert [22]

System Knowledge. Has high system knowledge [35]

Domain Knowledge. Has high domain knowledge [36];

knows accurate or precise information [33]

differences (e.g. cognitive ability) and experience in using the search system. Different tasks and searcher characteristics can be classified as leading to low or high ability to specify information need (see Table 1).

Table 1 shows examples of task characteristics (including characteristics of the information search process) and searcher characteristics (individual differences, information goal, levels of system and domain knowledge) which determine a searcher's ability to specify information need. E.g. using the classification of tasks by Spool et al. [32], a searcher dealing with judgment questions will have lower clarity of the task and a lower ability to specify information need, compared to when he is dealing with simple fact questions. Similarly, a student starting to work on a new project (with little domain knowledge) will have a lower ability to specify his information need appropriately, compared to another student who has been working on the project for 2 years.

Topical well-defined requests on content (only) is just one approach to document retrieval (more suited for a user with high ability to specify information need) albeit the most popular in IR research. A user with low ability to define information need may define requests vaguely. Also, requests may be non-topical (e.g. by journal or genre) and/or non-content based [42].

\section{Conclusion and Future Work}

In this study, we have proposed the 'searcher ability to specify his information need' as a solution to capturing the task/searcher characteristics. As per Dervin [6]'s sensemaking theory, the needs of searchers change continuously (a unique situation in time/space) and is very difficult to pin-point specifically from an IS perspective, because it resides in the searcher's head. However, operationalizing these complex 
constructs as the searcher's ability to specify information need is possible. Technology characteristics can be built to match this ability. This will help designers develop better interfaces for search, which will lead to greater human-computer interaction and search efficacy.

The study answers Kuhlthau [18]'s call for collaboration between the person and system-centered aspects of information seeking/retrieval. It also takes on the calls of Ingwersen and Jarvelin [19,20] by providing a way to include context and task in developing technology interfaces for search. The study will contribute to theory development in the field of HCI pertaining to information search and retrieval. The importance of this effort is highlighted by recent efforts such as the Information Retrieval in Context (IRiX) workshop of ACM SIGIR and the HARD track of the Text REtrieval Conference (TREC), that seek to leverage additional information about the searcher and his context to improve search accuracy.

From the practitioner's perspective, the study will be useful for developers of information systems for search - knowledge providers, content providers as well as designers of next-generation web search engines. As the organizers of IRiX 2005 mention in the preface of their proceedings, 'The underlying hypothesis (and belief) is that by taking context into account, the next generation of retrieval engines dependent on models of context can be created, designed and developed delivering performance exceeding that of out-of-context engines.' [43].

Future work on the study will include designing research studies utilizing the 'user ability to specify information need'. The relationship between user ability to specify need and technology characteristics will be investigated to test for fit.

\section{References}

1. Dix, A.: Human-Computer Interaction and Web Design. In: Proctor, R.W., Vu, K.-P.L. (eds.) Handbook of Human Factors in Web Design, pp. 28-47. Lawrence Erlbaum, Mahwah, New Jersey (2005)

2. Morahan-Martin, J., Anderson, C.D.: Information and Misinformation Online: Recommendations for Facilitating Accurate Mental Health Information Retrieval and Evaluation. CyberPsychology \& Behavior 3(5), 731-746 (2000)

3. Sullivan, D.: New Estimate Puts Web Size At 11.5 Billion Pages \& Compares Search Engine Coverage. SearchEngineWatch (May 17, 2005). Retrieved Feb 14, 2007 from http://blog.searchenginewatch.com/blog/050517-075657

4. Deerwester, S., Dumais, S.T., Furnas, G.W., Landauer, T.K., Harshman, R.: Indexing by latent semantic analysis. J. Society for Info. Sc. 41(6), 391-407 (1990)

5. Belkin, N.J.: Helping people find what they don't know. Communications of the ACM 43(8), 58-61 (2000)

6. Dervin, B.: From the mind's eye of the user: The sense-making qualitative-quantitative methodology. In: Glazier, J., Powell, R. (eds.) Qualitative research in information management. Libraries Unlimited, Englewood, CO, pp. 61-84 (1992)

7. Narayanan, S., Koppaka, L., Edala, N., Loritz, D., Daley, R.: Adaptive Interface for Personalizing Information Seeking. CyberPsychology \& Behavior 7(6), 683-688 (2004)

8. Dervin, B., Nilan, M.: Information needs and uses. In: Williams, M.E. (ed.) Annual review of Info. Sc. \& Tech. 21, pp. 3-33. Knowledge Industry Publ., White Plains, NY (1986) 
9. Belkin, N.J., Oddy, R., Brooks, H.: ASK for information retrieval. J. Documentation. 38(2), 61-71 (1982)

10. Ingwersen, P.: Information retrieval interaction. Taylor Graham, London (1992)

11. Mick, C.K., Lindsey, G.N., Callahan, D.: Toward usable user studies. JASIS. 31(5), 347 365 (1980)

12. Bystrom, K., Jarvelin, K.: Task complexity affects information seeking and use. Information Processing and Management. 31(2), 191-213 (1995)

13. Wilson, T.D.: Information needs and uses: Fifty years of progress? In: Vickery, B.C. (ed.) Fifty years of information progress: A J. Documentation review, Aslib, London, pp. 15-52 (1994)

14. Dervin, B.: An overview of sense-making research: concepts, methods and results to date. In: Int. Comm. Asso. Annual Meeting (1983)

15. Wilson, T.D.: Models in information behavior research. J. Documentation. 55(3), 249-270 (1999)

16. Kuhlthau, C.C.: Inside the search process: Information seeking from the user's perspective. JASIS 42(5), 361-371 (1991)

17. Savolainen, R.: Everyday life information seeking: approaching information seeking in the context of 'way of life'. LIBRES. 17(3), 259-294 (1995)

18. Kuhlthau, C.C.: Towards collaboration between information seeking and information retrieval. Information Research. 10, 2 (2005)

19. Ingwersen, P., Jarvelin, K.: The Turn: Integration of Information Seeking and Retrieval in Context Series. The Information Retrieval Series. 18(XIV), 448 (2005)

20. Jarvelin, K., Ingwersen, P.: Information seeking research needs extension towards tasks and technology. Information Research 10(1), 17 (2004)

21. Goodhue, D.L., Thompson, R.L.: Task-technology fit and individual performance. MIS Quarterly 19(2), 213-236 (1995)

22. Kuhlthau, C.C.: Seeking meaning: a process approach to library and information services, 2nd edn. Libraries Unlimited, Westport, CT (2004)

23. van Ossenbruggen, J., Hardman, L.: Smart Style on the Semantic Web. Semantic Web Workshop, WWW (2002)

24. Case, D.O.: Looking for information: A Survey of Research on Information Seeking, Needs, and Behavior. Academic Press, Elsevier, San Diego, CA (2002)

25. Kim, K.-S., Allen, B.: Cognitive and task influences on Web searching behavior. JASIST. 53(2), 109-119 (2002)

26. Saracevic, T., Kantor, P.: A study of information seeking and retrieving II. Users, questions, and effectiveness. JASIS 39(3), 177-196 (1988)

27. Marchionini, G.: Information seeking strategies of novices using a full-text electronic encyclopedia. JASIS 40(1), 54-66 (1989)

28. Qiu, L.: Analytical searching vs. browsing in hypertext information retrieval systems. Canadian J. Info. and Lib. Sc. 18(4), 1-13 (1993)

29. Kim, K.-S.: Users, tasks and the Web: Their impact on the information-seeking behavior. In: Proc. 21st national online meeting, pp. 189-198 (2000)

30. Matthews, J.R., Lawrence, G.S., Ferguson, D.K.: Using online catalogs: A nation wide survey. Neal-Schumann, New York (1983)

31. Pfaffenberger, B.: Web search strategies. MIS Press, New York (1996)

32. Spool, J., Scanlon, T., Snyder, C., DeAngelo, T.: Web site usability: A designer's guide. Morgan Kaufmann, San Francisco (1999)

33. Bhavani, S.K., Drabenstott, K., Radev, D.: Toward a Unified Framework of IR Tasks and Strategies. Proc. ASIS Annual Meeting 38, 340-354 (2001) 
34. Limberg, L.: Information use for learning purposes. In: Vakkari, P., Savolainen, R., Dervin, B. (eds.) Proc. ISIC 1996, pp. 275-289. Taylor Graham, London (1997)

35. Wildemuth, B.M.: The Effects of Domain Knowledge on Search Tactic Formulation. JASIST. 55(3), 246-258 (2003)

36. Miura, A., Fujihara, N., Yamashita, K.: Retrieving information on the World Wide Web: Effects of domain specific knowledge. AI and Society. 20(2), 221-231 (2006)

37. van de Wijngaert, L.: A policy capturing study of media choice: The effect information [sic] of needs and user characteristics on media choice. In: Wilson, T.D., Allen, D.K. (eds.) Information behavior: Proc. ISIC 1998, Taylor Graham, London, pp. 463-478 (1999)

38. Harter, S.P.: Psychological relevance and information science. JASIS 43, 602-615 (1992)

39. Ingwersen, P., Jarvelin, K.: Information Retrieval in Context. In: Proc. SIGIR IRiX Workshop, pp. 6-9 (2004)

40. Hawking, D., Paris, C., Wilkinson, R., Wu, M.: Context in Enterprise Search and Delivery. In: Proc. SIGIR 2005 IRix Workshop, pp. 14-16 (2005)

41. Green, A.: What do we mean by user needs? British J. Academic Librarianship 5, 65-78 (1990)

42. Jarvelin, K., Ingwersen, P.: Extending Information Seeking and Retrieval Research toward Context. In: Proc. SIGIR 2004 IRiX Workshop, pp. 11-15 (2004)

43. Ingwersen, P., Jarvelin, K., Belkin, N.: Proc. SIGIR IRiX Workshop (2005) 